\title{
Drainage patterns network and hydrogeological functions, Wadi El Atfehy hydrographic basin, Eastern Desert, Egypt
}

\author{
Samah Mahmoud Morsy Saad* \\ Department of Geology (Hydrogeology), Faculty of Science, Ain Shams University, Cairo, 11566, Egypt
}

A R T I C LE INFO

Article history:

Received 02 April 2016

Accepted 15 June 2016

Keywords:

Wadi El Aftehy;

Eastern Desert, Egypt;

Hydrographic basin;

Drainage network;

Oro-graphic characteristics.

\begin{abstract}
A B S T R A C T
The present work deals with the morphometric analysis of the hydrographic drainage network of Wadi El Aftehy hydrographic basin in the Eastern Desert of Egypt in order to investigate the impacts on the hydrogeologic response .The drainage network is expressed by several parameters comprising three aspects; linear, aerial and relief aspects. They control the behavior of the catchment with the occasional heavy showers during rainfall storms, surface runoff and infiltration possibilities. Techniques for minimizing flash flood hazardous and maximizing available water harvesting and groundwater recharge are focused. In spite of the prevailed desert conditions of arid climate, the well developed Orographic characteristics receiving the occasional rainfall storms causing torrential flash floods, possibly once over some years during winter season. The obtained results of the parameters of the drainage network and the structural lineation revealed high possibilities for draining, harvesting and infiltration downward for the surface runoff water during flash floods in order to recharge the groundwater aquifers. Estimating the hazard degrees indicates sub-basins having variable measures. The most hazardous ones are located in the middle part of the hydrographic basin. Proper techniques for sustainable development of both water and land resources in Wadi El Atfehy hydrographic basin are presented.
\end{abstract}

\section{Introduction}

Wadi El Atfehy hydrographic basin occupies an area of about $450 \mathrm{Km}^{2}$.The upstream part of the basin is located at the western part of the northern Galala Plateau, and it debouches into the Nile Valley. It is bounded by latitudes $29^{\circ} 10^{\prime}-29^{\circ} 30^{\prime} \mathrm{N}$ and longitudes $31^{\circ} 10^{\prime}-31^{\circ} 50^{`}$ E. (Fig. 1).

The distribution of rainfall along the higher ranges of Northern Galala Plateau and the slopes due east and west is naturally controlled by the Oro-graphic network. High mountainous relief, variable slopes, meandering courses, scattered boulders and sparse vegetation characterize the hydrographic basin. In spite of the arid conditions prevailed, it received an occasional high rainfall storms during the last decade. The resulted flash floods with great hazards are recorded either on the surface soils or on foundations installed at the downstream parts.

The hydrographic basin is mainly developed through the Eocene carbonate rocks. Upper- Middle Eocene rock units are exposed on the surface and covered by quaternary deposits within the main channel, tributaries and the delta ${ }^{[1-2]}$.

\footnotetext{
* Corresponding author.

E-mail address: samorsy@gmail.com
}

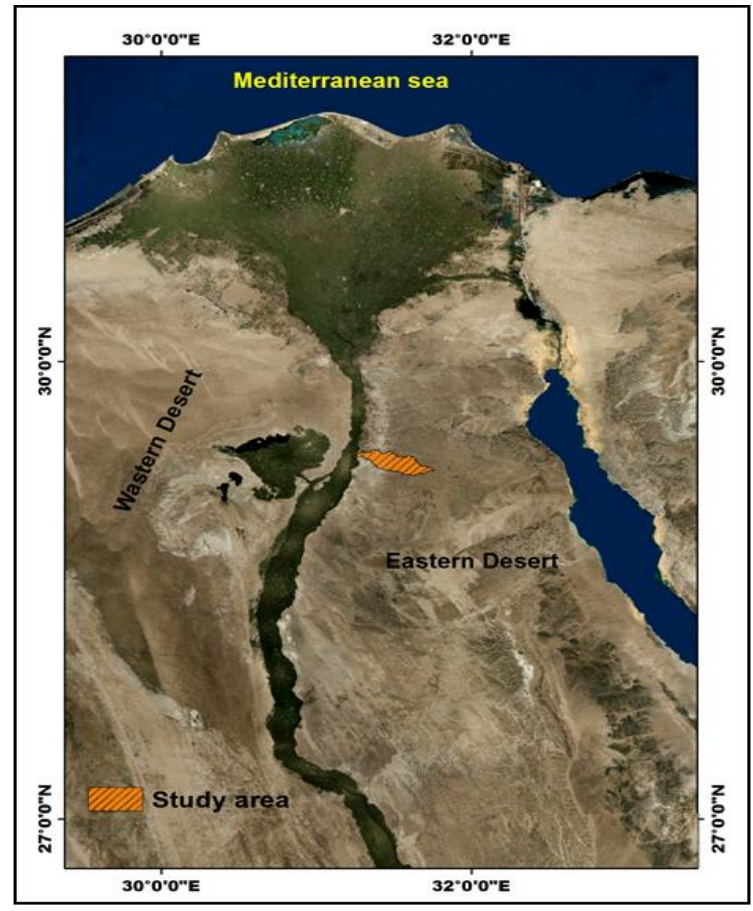

Fig (1): Location map of wadi El-Atfehy hydrographic basin, Eastern Desert, Egypt. 
Two aquifer systems are defined by previous works in El-Atfehy hydrographic basin; the Quaternary aquifer occupying the downstream and delta parts and formed of unconsolidated gravels, sands and clay intercalations and Middle Eocene aquifer occupying the upstream and mid-stream areas, built of limestone and chalky limestone water bearing rocks. They are mainly recharged by rainfall during the occasional storms, lateral inflow from the connected aquifers in the neighboring basins and in the Nile Valley ${ }^{[2-3]}$.

The drainage network has been developed through Carbonate terrain with variable features associated with the denudations and modification by surface and groundwater flows. Paleo-karstified features are recorded such as; the wide and narrow caves, the shallow and deep meanders and highly rugged channels. Many destructive and constructive landforms are developed due to the torrential floods ${ }^{[4-5]}$.

\section{Materials and Methods}

The ASTER satellite image, digital elevation model (DEM), ArcGIS software, Watershed Modeling System (WMS) and topographic maps represent the main techniques that used to achieve the aim of the present work.
Watershed modeling system (WMS 8.3 software) is used to delineate watersheds in the study area.

Aster Dem image is used to obtain the drainage pattern and to delineate the sub- basins.

The topographic maps are used to determine the streams junction and the outlets of sub-basins.

The ArcGIS Spatial Analyst toolbar (Hydrology toolset, ArcGIS 10.1 software), and DEM are used to obtain the flow directions, flow accumulation, stream-links, stream orders, and watersheds boundaries. Strahler's system of stream analysis is probably the simplest and most used system is adopted for the present study.

About 17 parameters are defined by the present work. They are classified into Three Aspects; Linear Aspect (including 4 parameters), Areal Aspect (including 9 parameters) and Relief Aspect (including 4 parameters). They are computed using standard methods and formulae (Table 1).

Evaluation of flash floods hazards are carried out through the calculation of hazard degree for the morphometric parameters that have a direct effect on flooding in the Wadi ElAtfehy hydrographic basin.

Table 1: Formulae for the computation of morphometric parameters.

\begin{tabular}{|c|c|c|}
\hline S. No. & Morphometric Parameters & Formula/Definition \\
\hline 1 & Stream order & Hierarchical Rank \\
\hline 2 & Bifurcation Ratio (Rb) & $\begin{array}{l}\mathrm{Rb}=\mathrm{Nu} / \mathrm{Nu}+1 \mathrm{Where}, \mathrm{Nu}=\mathrm{Number} \text { of stream segments present } \\
\text { in the given orderNu+1= Number of segments of the next higher } \\
\text { order }\end{array}$ \\
\hline 3 & Mean Bifurcation Ratio (Rbm) & Rbm $=$ Average of bifurcation ratios of all orders \\
\hline 4 & Stream Length $(\mathrm{Lu})$ & Length of the Stream $(\mathrm{km})$ \\
\hline 5 & Mean Stream Length (Lsm) & $\begin{array}{l}\mathrm{Lsm}=\mathrm{Lu} / \mathrm{Nu}, \mathrm{km} \text { Where, } \mathrm{Lu}=\text { Mean stream length of a given } \\
\text { order }(\mathrm{km}) \mathrm{Nu}=\text { Number of stream segments }\end{array}$ \\
\hline 6 & Stream Length Ratio (RL) & $\begin{array}{l}\mathrm{RL}=\mathrm{Lu} / \mathrm{Lu}-1 \text { Where, } \mathrm{Lu}=\text { Total stream length of order }(\mathrm{u}) \mathrm{Lu}- \\
1=\text { The total stream length of its next lower order }\end{array}$ \\
\hline 7 & Drainage Density (D) & $\begin{array}{l}\left.\mathrm{D}=\sum \mathrm{Lu} / \mathrm{Au}\right) \mathrm{km} / \mathrm{km} 2 \text { Where,Lu=Total Stream length of all } \\
\text { orders }(\mathrm{km}) \mathrm{Au}=\text { Area of the Basin }\left(\mathrm{km}^{2}\right)\end{array}$ \\
\hline 8 & Drainage Texture (Rt) & $\mathrm{Rt}=\sum \mathrm{Nu} / \mathrm{P}$ Where, $\mathrm{Nu}=$ Stream Number $\mathrm{P}=$ Perimeter $(\mathrm{km})$ \\
\hline 9 & Stream Frequency (Fs) & $\begin{array}{l}\mathrm{Fs}=\sum \mathrm{Nu} / \mathrm{Au} \text { Where, } \mathrm{Nu}=\text { Total number of streams in the basin } \\
\mathrm{Au}=\mathrm{Basin} \text { Area }\left(\mathrm{km}^{2}\right)\end{array}$ \\
\hline 10 & Infiltration No. (If) & If $=\mathrm{Rt} *$ Fs Where, $\mathrm{Rt}=$ Drainage Texture Fs $=$ Stream Frequency \\
\hline 11 & Length of Over Land Flow (Lg) & $\mathrm{Lg}=1 / \mathrm{D} \times 2 \mathrm{Km}$ Where, $\mathrm{D}=$ Drainage density $\left(\mathrm{km} / \mathrm{km}^{2}\right)$ \\
\hline 12 & Form Factor (Rf) & $\begin{array}{l}\mathrm{Rf}=\mathrm{Au} / \mathrm{Lb}^{2} \text { Where, } \mathrm{Au}=\text { Area of the Basin }\left(\mathrm{km}^{2}\right) \mathrm{Lb}=\text { Maximum } \\
\text { Basin length }(\mathrm{km})\end{array}$ \\
\hline 13 & Circularity Ratio (Rc) & $\begin{array}{l}\mathrm{Rc}=4 \pi \mathrm{Au} / \mathrm{P}^{2} \text { Where, } \mathrm{Au}=\text { Basin Area }\left(\mathrm{km}^{2}\right) \mathrm{P}=\text { Perimeter of } \\
\text { the basin }(\mathrm{km}) \Pi=3.14\end{array}$ \\
\hline 14 & Elongation Ratio (Re) & $\begin{array}{l}\mathrm{Re}=\sqrt{\mathrm{Au}} / \pi / \mathrm{Lb} \text { Where, } \mathrm{Au}=\text { Area of the Basin }\left(\mathrm{km}^{2}\right) \\
\mathrm{Lb}=\text { Maximum Basin length }(\mathrm{km}) \Pi=3.14\end{array}$ \\
\hline 15 & Relief Ratio (Rh) & $\begin{array}{l}\mathrm{Rh}=\mathrm{H} / \text { Lbmax Where, } \mathrm{H}=\text { Maximum basin relief }(\mathrm{km}) \text { Lbmax }= \\
\text { Maximum basin length }(\mathrm{km})\end{array}$ \\
\hline 16 & Ruggedness Number (HD) & $\begin{array}{l}\mathrm{HD}=\mathrm{H} \times \mathrm{Dd} \text { Where, } \mathrm{H}=\text { Maximum basin relief } \mathrm{Dd}=\text { Drainage } \\
\text { density }\end{array}$ \\
\hline 17 & Relative Relief (Rhp) & $\begin{array}{l}\text { Rhp }=\mathrm{H} \times(100) / \mathrm{P} \text { Where, } \mathrm{H}=\text { Maximum basin relief } \mathrm{P}= \\
\text { Perimeter of the basin }(\mathrm{km})\end{array}$ \\
\hline
\end{tabular}




\section{Results and Discussion}

The defined parameters are classified and computed numerically into Three Aspects as following ;

1- The first Aspect deals with linear morphometric parameters. It includes the following; Stream order (U), Stream Number $(\mathrm{Nu})$, Bifurcation Ratio $(\mathrm{Rb})$, and Stream Length $(\mathrm{Lu})$, The stream orders of the drainage basin plays first step in analysis of drainage basin ${ }^{[6]}$.

2- The second Aspect deals with the Areal morphometric parameters. It includes the following; Basin Area, Drainage Density, Stream Frequency, Infiltration Number (If), Drainage Texture, Length of Overland Flow, Drainage Texture (Rt), Circularity Ratio (Rc), Elongation Ratio (Re).

3- The third Aspect deals with the relief morphometric parameters. It includes the following; Relief Ratio, Relative Relief ratio, Ruggedness Number, and Basin Slope".

Wadi El Atfehy hydrographic basin is divided into sixteen sub-basins (Fig. 2). They are classified into four main regions starting from the upstream to the downstream respectively (W1, W2, W3 and W4) (Fig. 3).

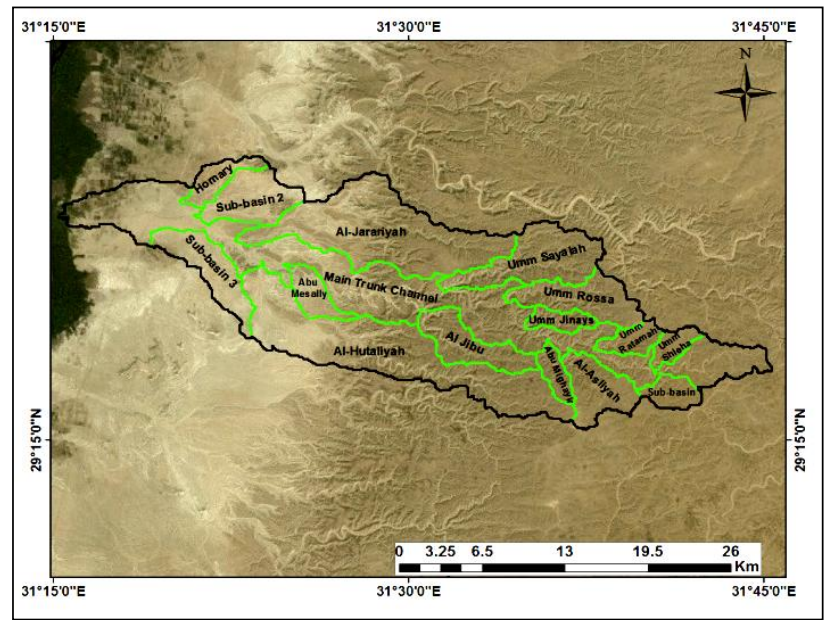

Fig (2): Wadi El-Atfehy hydrographic sub-basins, Eastern Desert, Egypt.

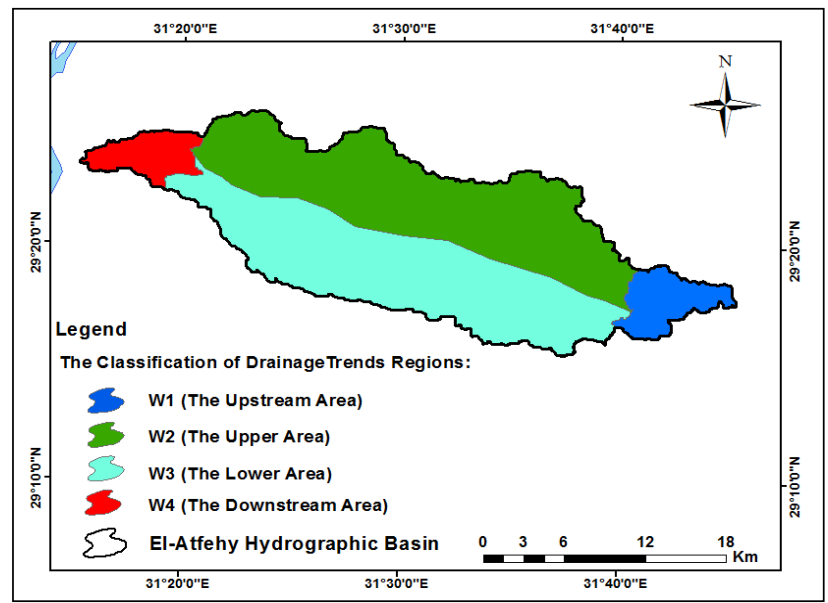

Fig (3): Classification of Wadi EL Atfehy hydrographic basins into four main regions according to the analysis of drainage trends.
The drainage networks and structural lineation in the defined Four regions are subjected to the analyses. The obtained results are represented by Figures $4 \mathbf{a} \& \mathbf{b}$ and correlated.

The first region in the upstream area (W1) include the following three subbasins; Eastern part of main trunck channel, Umm Shieha and sub-basin1. The second region in the upstream area (W2) include the following eight sub-basins; Northern part of main trunck channel, Umm Rossa, Umm Sayalah, Umm Jinays, Umm Ratama, Al-Jarariyyah, Sub-basin 2 and Homary. The third region in the downstream area (W3) include the following seven sub-basins; the Southern part of main trunck channel, Al-Asliyyah, Abu Mighayir, Al-Jibu, AlHutaliyyah, Abu Mesally, and Sub-basin3. The fourth region in the downstream area (W4) includes the Western part of main trunck channel subbasin.

It is obvious that the drainage streams in the regions W1 and W2 (Figs. 4 a $\&$ b) have the same trends of the structural lineations. This confirms the effective control of the geologic structures upon the development of drainage networks in these sub-basins. While in W3 and W4 regions different trends are defined and the drainage streams reflect the influence of surface slope. The results are confirmed by using rose diagrams (Figs. 5a,5h, inclusive).

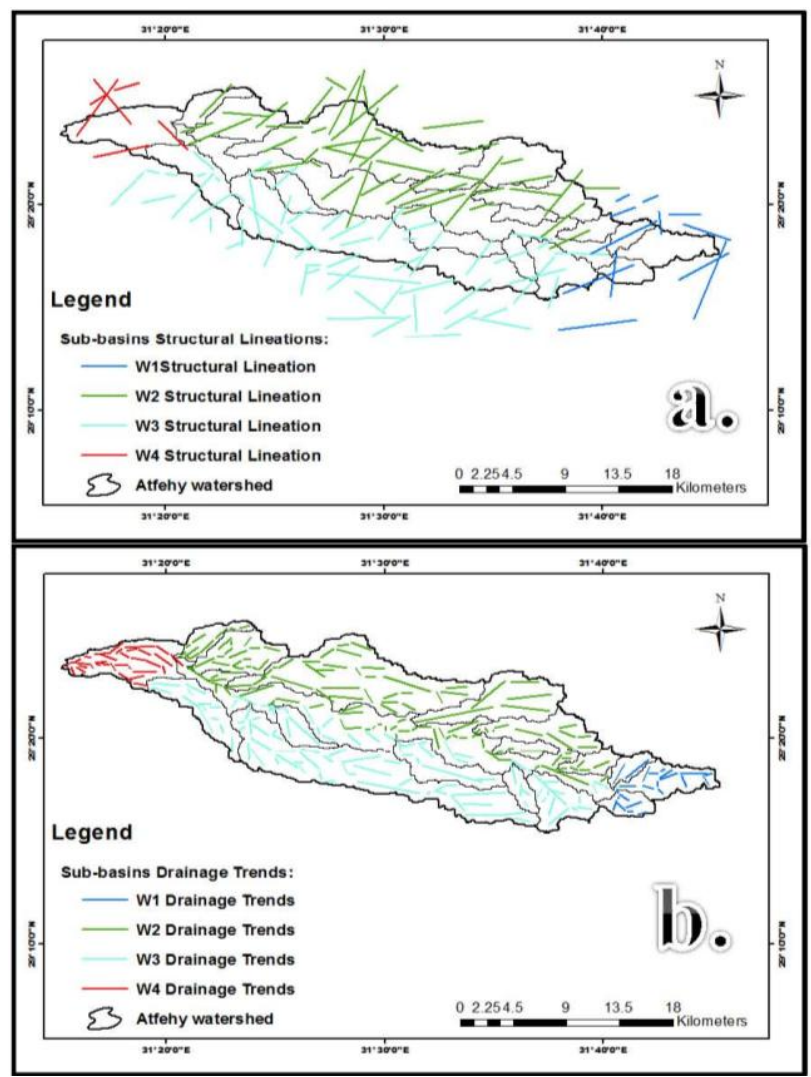

Fig (4): Drainage trends and structural lineation, Wadi El Atfehy hydrographic basin, Eastern Desert, Egypt.

The Following results and functions are obtained by the morphometric analysis of Wadi El-Atfehy hydrographic basin. They are classified into the three aspects as follows: 


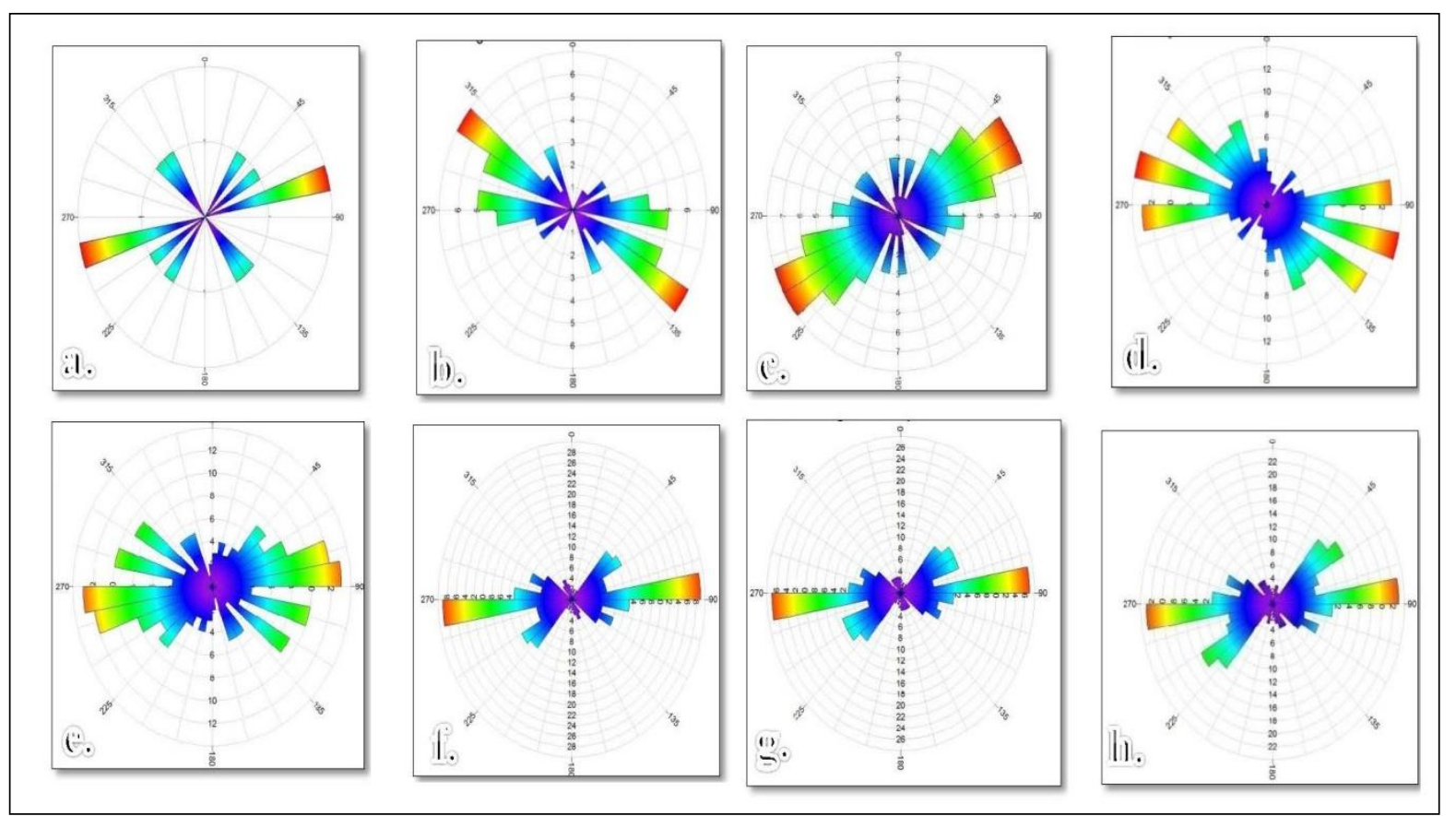

Fig (5): Rose diagrams of drainage trends and structural lineation trends, Wadi El Atfehy hydrographic basin, Eastern Desert, Egypt. $(\mathrm{a}, \mathrm{b})$ for region $\mathrm{W} 1,(\mathrm{c}, \mathrm{d})$ for region $\mathrm{W} 2,(\mathrm{e}, \mathrm{f})$ for region $\mathrm{W} 3,(\mathrm{~g}, \mathrm{~h})$ for region $\mathrm{W} 4$.

\section{Linear aspect (Table 2)}

The hydrographic basin has a $6^{\text {th }}$ stream order characteristics (Fig. 6). The values of bifurcation ratios $(\mathrm{Rb})$ of the subbasins are moderate to high indicating a region of steeply dipping rock strata. There is a direct relationship between the bifurcation ratio and both the surface water discharge and time of concentration. The bifurcation ratio decreases with the decrease of the time of arrival of water to the outlet of the wadi and vice versa.

The main trunck channel and Al-Hutaliyyah subbasins have high bifurcation ratio and they have an elongated shape .They yield low discharge but extended peak of flow which permits downward percolation of runoff water to contribute the groundwater aquifers.

The mean bifurcation ratio of each sub-basin in the study area lies within the standard ranges and indicates

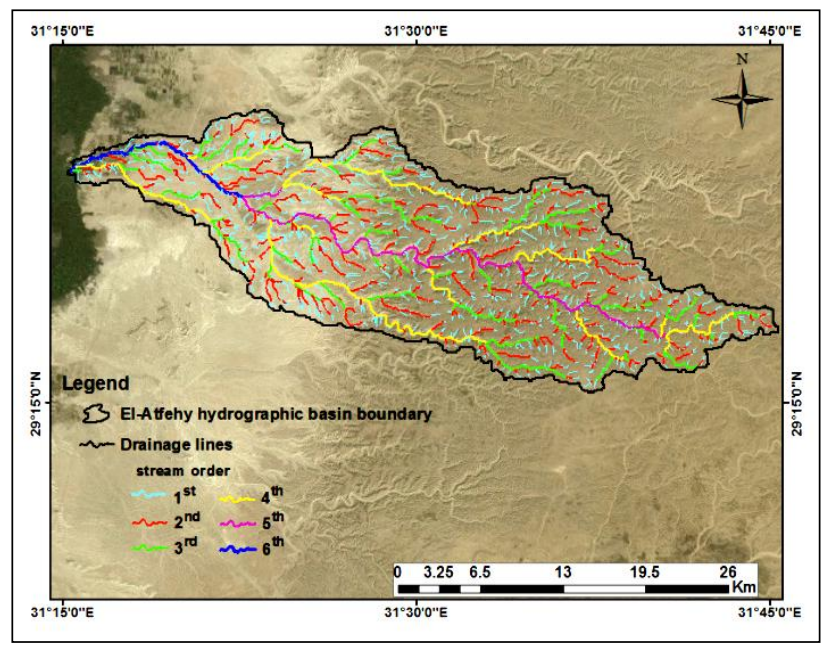

Fig (6): Stream orders, Wadi El-Atfehy hydrographic subbasins, Eastern Desert, Egypt. that all subbasins have the characteristics of natural streams; reflecting high influence of geological structures on the drainage pattern.

The relation between stream order (U) and stream length (Lu) is illustrated by Fig. 7. It indicates that the streams of relatively smaller lengths have areas with larger slopes and finer textures. While, longer lengths of stream are generally indicate flatter gradients. Generally, the total length of stream segments is the maximum for first order streams and decreases as the stream order increases. In other words, the stream lengths are decreasing with the increasing stream orders.

It becomes obvious that, the downstream area of the basin has low values of length of overland flow (lg) indicating that; the surface water is accumulated at this area faster than the other areas of high values of $(\mathrm{lg})$. It is less eroded area; where it has a lower length of overland flow.

\section{Areal aspects (Table 3)}

The basin length $(\mathrm{Lb})$ reflects the travel time of surface runoff especially the flood flow through the basin. The longest basin length represents high potentiality of groundwater recharge than the shortest travel basin length. The basin length in most sub-basins of the study area has lower values; indicating short travel time and low potentiality of groundwater recharge. At the middle area of the basin, the sub-basins have great value (between $19-42 \mathrm{Km}$ ) indicating high potential recharge to groundwater.

Horton ${ }^{[7]}$ inferred that the mean drainage basin areas of progressively higher orders should increase in a geometric sequence, as do stream lengths. The area of the basin $(\mathrm{Au})$ is defined as the total area projected upon a horizontal plane contributing to cumulate of all basin orders. 
Table 2: Values of linear aspects, Wadi El Atfehy hydrographic sub-basin, Eastern Desert, Egypt.

\begin{tabular}{|c|c|c|c|c|c|c|c|c|c|c|c|c|c|c|c|c|}
\hline $\begin{array}{l}\text { stream } \\
\text { order } \\
(\mathrm{Nu})\end{array}$ & 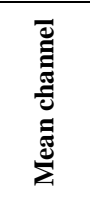 & 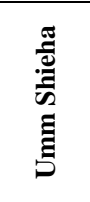 & 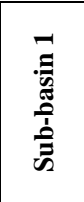 & 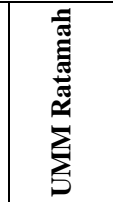 & $\frac{\substack{\frac{\pi}{\pi} \\
\frac{\pi}{4}}}{4}$ & 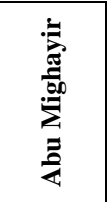 & 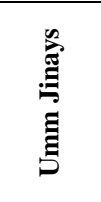 & 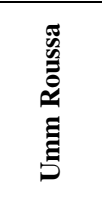 & 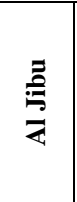 & 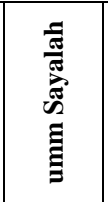 & 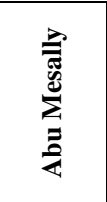 & 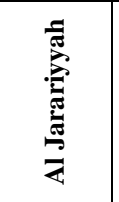 & 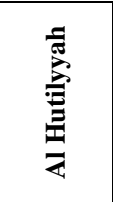 & 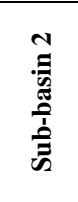 & 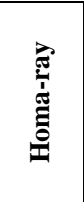 & 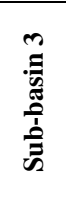 \\
\hline $1^{\text {st }}$ & 432 & 28 & 34 & 28 & 86 & 28 & 18 & 84 & 77 & 115 & 42 & 277 & 322 & 98 & 37 & 99 \\
\hline $2^{\text {nd }}$ & 85 & 6 & 4 & 6 & 18 & 8 & 4 & 15 & 14 & 14 & 9 & 45 & 52 & 16 & 5 & 16 \\
\hline $3^{\text {rd }}$ & 15 & 2 & 2 & 1 & 3 & 1 & 1 & 2 & 3 & 3 & 2 & 7 & 8 & 6 & 2 & 3 \\
\hline $4^{\text {th }}$ & 1 & 1 & 1 & - & 1 & - & - & 1 & 1 & 1 & 1 & 2 & 1 & 1 & 1 & 1 \\
\hline $5^{\text {th }}$ & - & - & - & - & - & - & - & - & - & - & - & 1 & - & - & - & - \\
\hline total & 535 & 37 & 41 & 25 & 108 & 37 & 23 & 102 & 94 & 133 & 54 & 332 & 383 & 121 & 45 & 119 \\
\hline $\begin{array}{c}\text { Bifurcatio } \\
\text { n ratio } \\
\text { (Rb) }\end{array}$ & 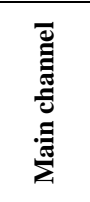 & 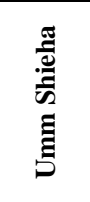 & 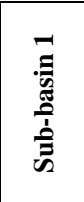 & 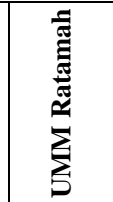 & $\begin{array}{l}\frac{\pi}{\pi} \\
\frac{5}{5} \\
\frac{\pi}{4}\end{array}$ & 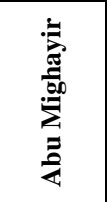 & 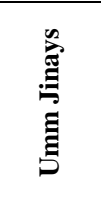 & 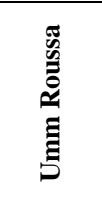 & $\frac{\Xi}{2}$ & 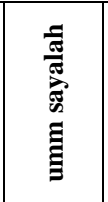 & 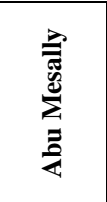 & 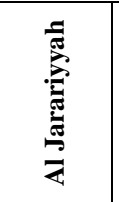 & 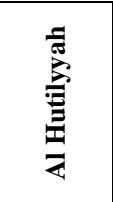 & 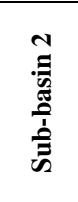 & 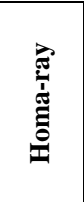 & 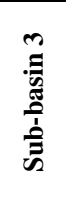 \\
\hline $1^{\text {st }} / 2^{\text {nd }}$ & 5.081 & 4.666 & 8.5 & 4.666 & 4.777 & 3.5 & 4.5 & 5.6 & 5.5 & 8.214 & 4.666 & 6.155 & 6.1923 & 6.125 & 7.4 & 6.18 \\
\hline $2^{\text {nd }} / 3^{\text {rd }}$ & 5.666 & 3 & 2 & 6 & 6 & 8 & 4 & 7.5 & 4.66 & 4.666 & 4.5 & 6.428 & 6.5 & 2.66 & 2.5 & 5.33 \\
\hline $3^{\text {rd }} / 4^{\text {th }}$ & 15 & 2 & 2 & - & 3 & - & - & 2 & 3 & 3 & 2 & 3.5 & 8 & 6 & 2 & 3 \\
\hline & & - & - & - & - & - & - & - & - & - & - & 2 & - & - & - & - \\
\hline total & 25.749 & 9.666 & 12.5 & 10.667 & 13.777 & 11.5 & 8.5 & 15.1 & 13.1 & 15.880 & 11.166 & 18.084 & 20.692 & 14.79 & 11.9 & 14.5 \\
\hline mean & 6.43 & 2.416 & 3.125 & 3.556 & 3.444 & 3.833 & 2.833 & 3.775 & \begin{tabular}{|l|}
3.29 \\
\end{tabular} & 3.970 & 2.791 & 3.616 & 5.173 & 3.697 & 2.975 & 3.63 \\
\hline $\begin{array}{c}\text { stream } \\
\text { Length } \\
\text { (Lu) Km. }\end{array}$ & 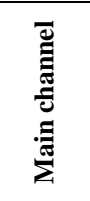 & 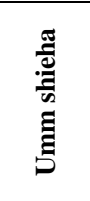 & 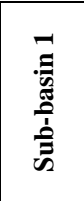 & 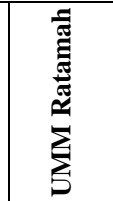 & 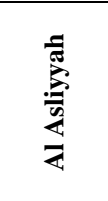 & 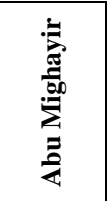 & 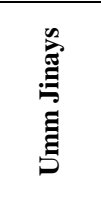 & 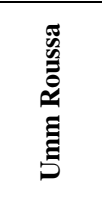 & $\frac{z}{3}$ & 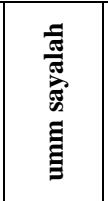 & 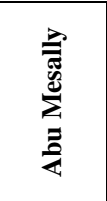 & 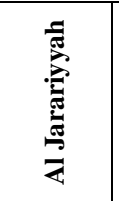 & 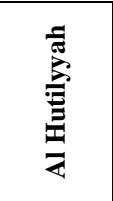 & 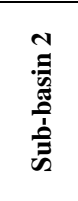 & 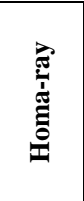 & 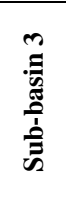 \\
\hline $1^{\text {st }}$ & 109.5 & 6.384 & \begin{tabular}{|l|}
6.961 \\
\end{tabular} & 8.306 & 22.2776 & 5.20369 & 6.20970 & 21.2683 & \begin{tabular}{|l|}
18.5 \\
\end{tabular} & 28.453 & 17.1108 & \begin{tabular}{|l|l|}
94.5312 \\
\end{tabular} & 89.3618 & 31.64 & 13.61 & 28.9 \\
\hline $2^{\text {nd }}$ & 56.67 & 1.824 & 5.062 & 3.893 & 10.4547 & 3.72425 & 3.45569 & 11.7055 & 8.76 & 13.250 & 6.14650 & \begin{tabular}{|l|l|}
33.3674 \\
\end{tabular} & 47.3348 & 9.567 & 4.860 & 10.4 \\
\hline $3^{\text {rd }}$ & 17.61 & 3.893 & 3.543 & 4.077 & 3.20988 & 4.13230 & 3.25454 & 3.11973 & 7.11 & 13.450 & 2.83386 & 17.1044 & 23.8139 & 8.076 & 5.200 & 8.99 \\
\hline $4^{\text {th }}$ & 6.310 & 1.437 & 1.351 & - & 5.73832 & - & - & 8.26731 & 3.83 & 7.2828 & 2.32058 & 20.2756 & 23.1254 & 5.619 & $\mathbf{0 . 7 8 0}$ & 4.56 \\
\hline $5^{\text {th }}$ & - & - & - & - & - & - & - & - & - & - & - & 3.24712 & - & - & - & - \\
\hline $6^{\text {th }}$ & - & - & - & - & - & - & - & - & - & - & - & - & - & - & - & - \\
\hline total & 236.1 & 13.539 & 16.91 & 16.277 & 41.6805 & 13.0602 & 12.9199 & 44.3609 & 34.4 & 62.437 & 28.4117 & 168.525 & 183.636 & 54.90 & 24.46 & 52.9 \\
\hline $\begin{array}{c}\text { Mean } \\
\text { stream } \\
\text { Length } \\
\text { (Lum) } \\
\text { Km. }\end{array}$ & 焉离 & 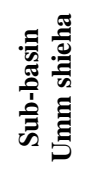 & 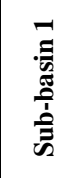 & 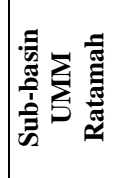 & 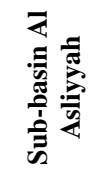 & 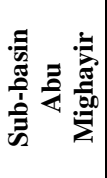 & 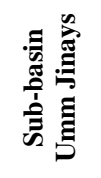 & 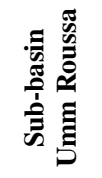 & 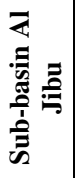 & 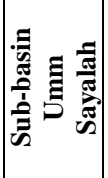 & 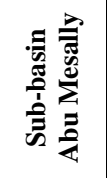 & 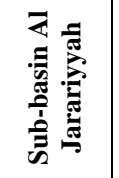 & 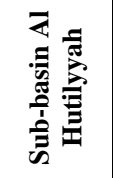 & 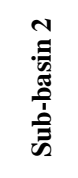 & 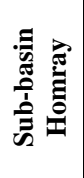 & 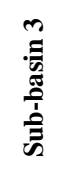 \\
\hline 1st & 0.253 & 0.228 & 0.2047 & \begin{tabular}{|l|}
0.29665 \\
\end{tabular} & 0.25904 & 0.18584 & 0.34498 & 0.253 & 0.240 & 0.2474 & 0.40740 & \begin{tabular}{|c|}
0.341268 \\
\end{tabular} & 0.277521 & 0.322 & 0.3680 & 0.292 \\
\hline 2nd & 0.666 & 0.304 & 1.2657 & \begin{tabular}{|l|}
0.64888 \\
\end{tabular} & 0.58081 & $\begin{array}{l}0.46553 \\
\end{array}$ & 0.86392 & 0.780 & 0.626 & \begin{tabular}{l|l|}
0.9464 \\
\end{tabular} & 0.68294 & \begin{tabular}{|l|}
0.741498 \\
\end{tabular} & 0.910284 & 0.597 & 0.9721 & 0.651 \\
\hline 3rd & 1.174 & 1.946 & 1.7717 & \begin{tabular}{|l|}
4.07788 \\
\end{tabular} & 1.06996 & 4.13230 & 3.25454 & 1.559 & 2.372 & 4.4834 & 1.41693 & 2.443490 & 2.976738 & 1.346 & 2.6004 & 2.997 \\
\hline 4th & 6.310 & 1.437 & 1.3515 & - & 5.73832 & - & - & 8.267 & 3.834 & \begin{tabular}{l|l|}
7.2828 \\
\end{tabular} & - & \begin{tabular}{l|l}
10.13780 \\
\end{tabular} & 23.12541 & 5.619 & 0.7809 & 4.564 \\
\hline 5th & - & - & - & - & - & - & - & - & - & - & - & 3.247126 & - & - & - & - \\
\hline 6th & - & - & - & - & - & - & - & - & - & - & - & - & - & - & - & - \\
\hline total & 54.42 & 3.916 & 4.5937 & 5.02342 & 7.64814 & 4.78367 & 4.46345 & 10.86 & 7.074 & 12.960 & 4.82786 & 16.91118 & 27.28995 & 7.886 & 4.7216 & 8.506 \\
\hline $\begin{array}{l}\text { stream } \\
\text { Length } \\
\text { ratio (RI) }\end{array}$ & 焉 & 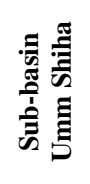 & 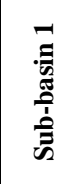 & 旗 & 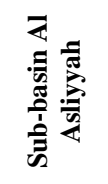 & 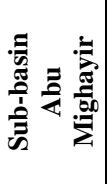 & 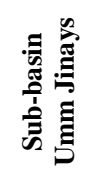 & 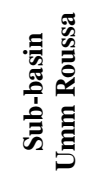 & 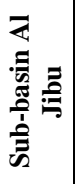 & 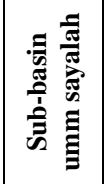 & 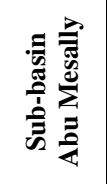 & 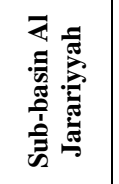 & 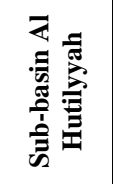 & 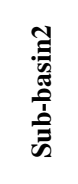 & 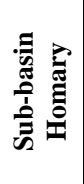 & 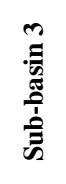 \\
\hline 2nd /1st & 0.517 & 0.285 & 0.7272 & \begin{tabular}{|l|l|} 
\\
\end{tabular} & 0.46929 & 0.71569 & 0.55649 & 0.550 & 0.473 & 0.4656 & 0.35921 & 0.352977 & 0.529698 & 0.302 & 0.3568 & 0.359 \\
\hline 3rd /2nd & 0.310 & 2.134 & 0.6998 & 1.04740 & 0.30702 & 1.10956 & 0.94179 & 0.266 & 0.811 & 1.0151 & 0.46105 & 0.512608 & 0.503094 & 0.844 & 1.0700 & 0.862 \\
\hline 4th /3rd & 0.358 & 0.369 & 0.3814 & - & 1.78770 & - & - & 2.650 & 0.538 & 0.5414 & 0.81887 & 1.185400 & 0.971088 & 0.695 & 0.1501 & 0.507 \\
\hline 5th /4th & - & - & - & - & - & - & - & - & - & - & - & 0.160149 & - & - & - & - \\
\hline 6th $/ 5$ th & - & - & - & - & - & - & - & - & - & - & - & - & - & - & - & - \\
\hline total & 7.784 & 2.789 & 1.8085 & 1.51612 & 2.56402 & 1.82525 & 1.49829 & 3.466 & 1.823 & 2.0222 & 1.63914 & 2.211136 & 2.003881 & 1.842 & 1.5770 & 1.729 \\
\hline
\end{tabular}


Table 3: Areal and relief aspects, Wadi El Atfehy hydrographic sub-basin, Eastern Desert, Egypt.

\begin{tabular}{|c|c|c|c|c|c|c|c|c|c|c|c|}
\hline $\begin{array}{l}\text { Area of the } \\
\text { basin }(\mathbf{A u}) \\
\left(\mathrm{Km}^{2}\right)\end{array}$ & $\begin{array}{l}\text { Perimeter } \\
\text { of the } \\
\text { basin }(\mathbf{P}) \\
(\mathbf{k m})\end{array}$ & $\begin{array}{c}\text { Basin } \\
\text { length } \\
(\mathbf{L b})(\mathbf{k m})\end{array}$ & $\begin{array}{c}\text { Rorm } \\
\text { factor } \\
(\mathbf{R f})\end{array}$ & $\begin{array}{l}\text { Elongation } \\
\text { ratio (Re) }\end{array}$ & $\begin{array}{c}\text { Length of } \\
\text { overland } \\
\text { flow }(\mathrm{Lg}) \\
\mathrm{Km}\end{array}$ & $\begin{array}{c}\text { Ruggedness } \\
\text { number } \\
\text { (Rn) }\end{array}$ & $\begin{array}{c}\text { Basin relief } \\
\text { (R) }\end{array}$ & $\begin{array}{c}\text { Relative } \\
\text { Relief } \\
\text { (Rhp) }\end{array}$ & $\begin{array}{c}\text { Relief } \\
\text { ratio }(\mathbf{R r})\end{array}$ & $\begin{array}{l}\text { Infiltration } \\
\text { No. (If) }\end{array}$ & \\
\hline $\begin{array}{c}\text { Main } \\
\text { channel }\end{array}$ & 94.63127 & 164.62933 & 42.26096 & 0.05298536 & 0.259649252 & 0.200326939 & 22.8773364 & 0.571 & 0.3468397 & 0.013511 & 14.19306 \\
\hline $\begin{array}{c}\text { Umm } \\
\text { Shieha }\end{array}$ & 5.58066 & 15.41296 & 4.36394 & 0.29304075 & 0.610622933 & 0.206087052 & 4.039306226 & 0.098 & 0.6358285 & 0.022456 & 16.08553 \\
\hline Sub-basin 1 & 7.15191 & 14.58046 & 3.47772 & 0.59133395 & 0.867412162 & 0.211349558 & 3.677482316 & 0.087 & 0.5966889 & 0.025016 & 13.56221 \\
\hline Al Asliyyah & 16.51751 & 25.79876 & 6.29562 & 0.41674228 & $\begin{array}{l}0.728186935 \\
\end{array}$ & 0.198144101 & 5.548034832 & 0.14 & 0.5426617 & 0.022237 & 16.49939 \\
\hline $\begin{array}{c}\text { Abu } \\
\text { Mighayir }\end{array}$ & 5.8591 & 15.02571 & 4.8503 & 0.24906303 & 0.56294211 & 0.224318547 & 6.011737071 & 0.134 & 0.8918047 & 0.027627 & 14.07537 \\
\hline $\begin{array}{l}\text { Umm } \\
\text { Jinays }\end{array}$ & 5.7045 & 16.3135 & 5.04895 & 0.22377699 & 0.53360123 & 0.220763323 & 7.505952988 & 0.17 & 1.0420817 & 0.033670 & 9.131735 \\
\hline $\begin{array}{c}\text { Umm } \\
\text { Roussa }\end{array}$ & 19.7916 & 32.52596 & 9.47922 & 0.22026003 & 0.529391482 & 0.22307448 & 9.50297285 & 0.213 & 0.6548615 & 0.022470 & 11.55152 \\
\hline $\begin{array}{c}\text { Umm } \\
\text { Sayalah } \\
\end{array}$ & 28.53025 & 38.89432 & 9.14603 & 0.34106749 & 0.658763103 & 0.228472269 & 13.2513916 & 0.29 & 0.7456101 & 0.031707 & 10.20193 \\
\hline $\begin{array}{c}\text { Abu } \\
\text { Mesally }\end{array}$ & 8.47548 & 19.24817 & 6.32625 & 0.21177349 & 0.519092676 & 0.149154307 & $\mathbf{5 . 3 9 9 3 8 5 9 1 6}$ & 0.181 & 0.9403491 & 0.028610 & 21.35815 \\
\hline $\begin{array}{c}\text { Al } \\
\text { Jarariyyah } \\
\end{array}$ & 63.5932 & 62.07229 & 19.35064 & 0.16983219 & 0.464856712 & 0.188674899 & 15.43360674 & 0.409 & 0.6589091 & 0.021136 & 13.83513 \\
\hline $\begin{array}{c}\text { Al } \\
\text { Hutilyyah } \\
\end{array}$ & 72.50987 & 78.06987 & 24.06628 & 0.12519275 & 0.399115595 & 0.197428241 & 16.38654398 & 0.415 & 0.5315751 & 0.017244 & 13.37711 \\
\hline Sub-basin 2 & 18.36741 & 25.44767 & 7.69496 & 0.31019528 & 0.628241604 & 0.167252425 & 4.448914493 & 0.133 & 0.5226411 & 0.017284 & 19.69404 \\
\hline Homaray & 8.10395 & 20.21637 & 6.61743 & $\begin{array}{l}0.18506233 \\
\end{array}$ & 0.485252878 & 0.165644563 & 4.604918857 & 0.139 & 0.6875616 & 0.021005 & 16.76133 \\
\hline Sub-basin 3 & 18.2822 & 31.1731 & 9.68689 & 0.19483249 & 0.497897322 & 0.172544611 & 4.762231274 & 0.138 & 0.4426890 & 0.014246 & 18.86190 \\
\hline
\end{tabular}

\begin{tabular}{|c|c|c|c|c|c|}
\hline Basin Name & $\begin{array}{c}\text { Drainage density } \\
\text { (Dd) }\end{array}$ & $\begin{array}{c}\text { Drainage texture } \\
\text { (Rt) }\end{array}$ & $\begin{array}{c}\text { Stream frequency } \\
\text { (Fs) }\end{array}$ & $\begin{array}{c}\text { Circulation ratio } \\
\text { (Rc) }\end{array}$ & $\begin{array}{c}\text { Compactness ratio } \\
\text { (Sh) }\end{array}$ \\
\hline Main channel & 2.49919 & 3.249724 & 5.65352235 & 0.04385406 & 4.77523838 \\
\hline Umm shieha & 2.426159 & 2.40057 & 6.63004017 & 0.29505508 & 1.84097736 \\
\hline Sub-basin 1 & 2.365748 & 2.811982 & 5.7327343 & 0.4225413 & 1.58386237 \\
\hline UMM Ratamah & 2.400668 & 2.115681 & 5.1619449 & 0.3111780 & 1.79265008 \\
\hline Al Asliyyah & 2.523416 & 4.186247 & 6.5385157 & 0.3116998 & 1.79114903 \\
\hline Abu Mighayir & 2.22897 & 2.42446 & 6.3147367 & 0.32596135 & 1.75152743 \\
\hline Umm Jinays & 2.26468 & 1.409875 & 4.0319046 & 0.269223 & 1.92727432 \\
\hline Umm Roussa & 2.241403 & 3.135956 & 5.15370157 & 0.2349688 & 2.0627921 \\
\hline Al Jibu & 2.003888 & 3.186881 & 5.4729343 & 0.24795528 & 2.00822938 \\
\hline Umm Sayalah & 2.188449 & 3.419522 & 4.66171870 & 0.23687697 & 2.05465341 \\
\hline Abu Mesally & 3.352233 & 2.805461 & 6.37132056 & 0.28732608 & 1.86557397 \\
\hline Al Jarariyyah & 2.650061 & 5.348602 & 5.22068397 & 0.20730261 & 2.19633007 \\
\hline Al Hutilyyah & 2.532565 & 4.905861 & 5.28203953 & 0.14942382 & 2.58696214 \\
\hline Sub-basin 2 & 2.989493 & 4.754855 & 6.5877551 & 0.35623904 & 1.67544140 \\
\hline Homaray & 3.018511 & 2.225918 & 5.55284768 & 0.2490468 & 2.00382588 \\
\hline Sub-basin 3 & 2.897801 & 3.817391 & 6.50903853 & 0.23629754 & 2.05717099 \\
\hline
\end{tabular}

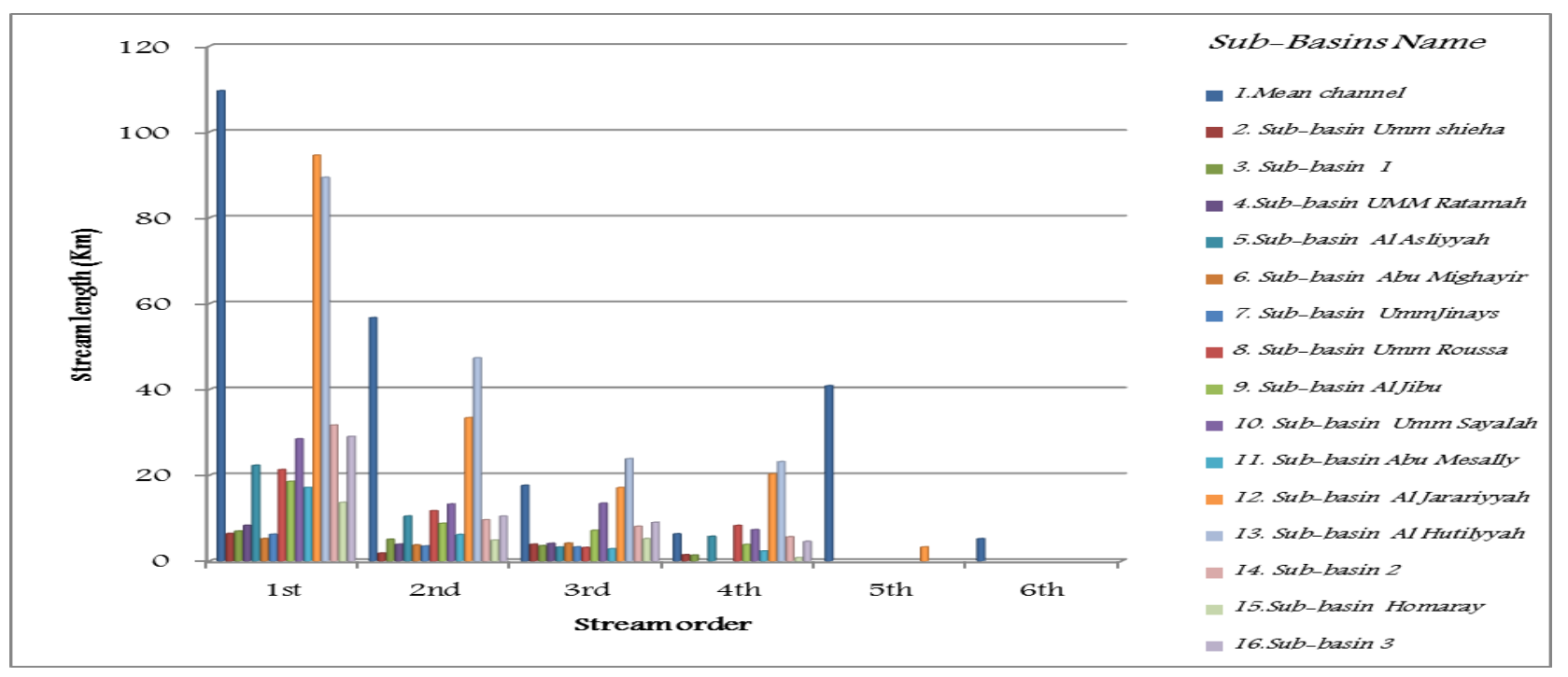

Fig (7): The relation between stream order and stream length, Wadi El-Atfehy hydrographic basins, Eastern Desert, Egypt. 
According to Gupta ${ }^{[\mathbf{8}]}$, compactness factor of the basin is used to express the basin shape, which is indicated by the deviation of the basin area from a circle having an equal area. A circular basin with low value of (Sh) is the most hazardous; because it will yield the shortest time of concentration before peak flow occurs in the basin. In Wadi El Atfehy hydrographic basin; the higher value of compactness ratio is recorded at the main trunck channel sub-basin indicating lowest hazardous area. According to Horton ${ }^{[9]}$, the form factor ( $\mathrm{Rf}$ ) is the ratio of the basin area to the square of the basin length. It is used as a quantitative expression of the shape of basin form, where Rf values of less than 5 reflecting that the subbasins have flat shape and longer durations of flow, enhancing the groundwater recharge possibilities and higher potentialities for runoff water harvesting. Rf values of greater than 5 reflecting high peaks and flow of shorter durations

The drainage density is an important indicator of the linear scale of landform elements in stream-eroded topography ${ }^{[7]}$. Thus the drainage density is simply the ratio of total channel-segment lengths cumulated for all orders within a basin to the basin area. The lowest values are favoured in regions of high resistant or high permeable sub soil materials, under dense vegetation cover and where the relief is low and also indicate most of rainfall infiltrates to recharge the groundwater. High drainage densities are favoured in regions of weak or impermeable surface materials, sparse vegetation, and mountainous relief. They indicate large volumes of the rainfall are converted into runoff. The drainage density is controlled by rock type, runoff intensity, soil type, and infiltration capacity of the soil. The higher values of drainage density are recorded in the following, Subbasin 2, Homaray, Sub-basin 3, Abu Mesally, Al Jarariyyah and Al Hutilyyah subbasins (Fig. 8). The value of stream frequency for the basin exhibits positive correlation with the drainage density value of the area; indicating faster runoff and therefore flooding is more likely in the basin ${ }^{[10]}$. The drainage texture is an important factor in the drainage morphometric analysis which depending on the underlying lithology ${ }^{[11]}$.

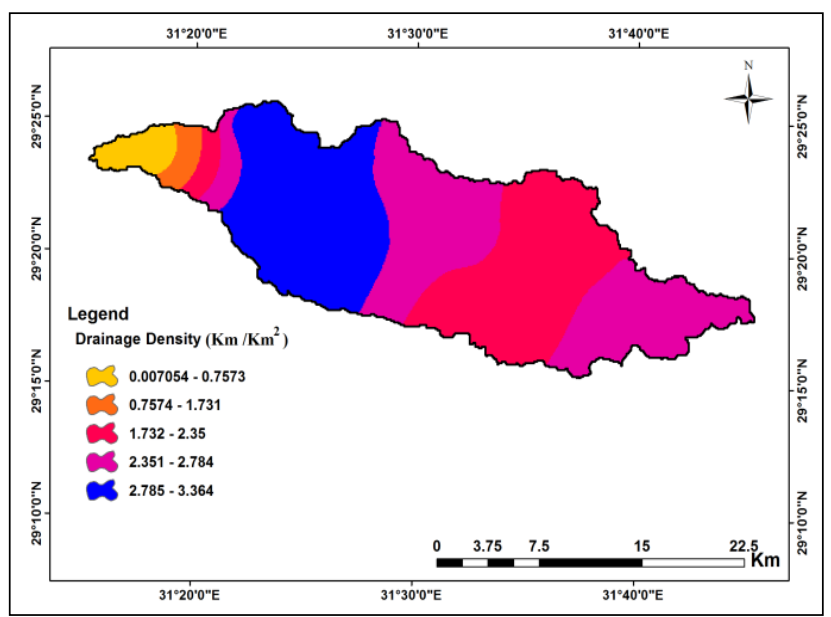

Fig (8): Areal distribution of the drainage density, Wadi ElAtfehy sub-basins, Eastern Desert, Egypt.
The lower values of drainage texture indicate that the basin has a good chance for groundwater recharge. The basins of high values are composed of hard rocks with no ability for water infiltration. They have a good chance to produce flash flood.

The drainage density and drainage frequency have been collectively defined as the drainage texture. The values of drainage basin texture $\left(R_{t}\right)$ are classified by Smith ${ }^{[12]}$ and Strahler ${ }^{[13]}$ into the following classes;

$$
\begin{array}{ll}
0-4 & \text { Coarse } \\
4-10 & \text { Intermediate } \\
10-15 & \text { Fine } \\
>15 & \text { Ultra Fine (bad land topography). }
\end{array}
$$

The obtained values of the drainage texture of the subbasins in the studied hydrographic basin lying between 1.4 and 5.3. This indicates an area of coarse to intermediate texture.

Miller ${ }^{[14]}$ described the basin of the circularity ratios ranging from 0.4 to 0.5 as a basin of strongly elongated and highly permeable homogenous geological materials. Circularity ratio $(\mathrm{Rc})$ is influenced by the length and frequency of streams, geological structures, land use/land cover, climate, relief and slope of the basin ${ }^{[15]}$.

The circularity ratios of the sub-basins in the studied hydrographic basin range from 0.04 to 0.43 . This indicates sub basins varying from oval to elongated shapes with homogenous geological materials. This ratio runs between 0.6 and 1.0 over a wide variety of climatic and geologic types ${ }^{[13]}$. The varying slopes of watershed can be classified with the help of the index of elongation ratio, i.e. circular (0.9-0.10), oval (0.8-0.9), less elongated (0.7-0.8), elongated (0.5-0.7), and more elongated $(<0.5)$.

The obtained elongation ratios range from 0.25 to 0.86 . This indicates that the sub-basins have an elongated to more elongated shape reflecting well generate low peak runoff and slower travel velocities to the outlet.

The infiltration number of a watershed is defined as the product of drainage density and stream frequency ${ }^{[16]}$. The higher the infiltration number, the lower will be the infiltration and the higher the surface runoff. Higher infiltration numbers are obtained for Sub-basin 2, Subbasin 3, Abu Mesally and Al Jarariyyah subbasins (Table 3).

The obtained values of the areal parameters such as drainage density, stream frequency, bifurcation ratio, drainage texture, and length of overland flow have a direct relationship with erodibility. The shape parameters such as elongation ratio, compactness coefficient, circularity ratio, basin shape and form factor have an inverse relationship with erodibility Where the lower the value, more is the erodibility.

\section{Relief aspects (Table 3)}

Difference in the elevation (R) between the highest topographic point of a watershed and the lowest one in the watershed known as the total relief of the river basin. The relief ratio $(\mathrm{Rr})$ may be defined as the ratio between the total relief of a basin and the longest dimension of 
the basin parallel to the main drainage line ranges from 0.0135 to 0.0336 , which indicates a low relief and moderate to gentle slope. The possibility of a close correlation between the relief ratio and the hydrologic characteristics of a basin is suggested by Schumm [11], $(\mathrm{Lu})$ is the maximum basin length.

Strahler ${ }^{[17]}$, defined the Ruggedness Number (Rn) as the product of maximum basin relief $(\mathrm{H})$ and drainage density (Dd), where both parameters are in the same unit. If drainage density is increased while the maximum basin relief remains constant, the average horizontal distance from divides to adjacent channels is reduced, with an accompanying increase in slope steepness. If drainage density is remains constant while the maximum basin relief increased, the elevation differences between divides and adjacent channels will also increase, so that the slope steepness increases. Extremely high values of the ruggedness number occure when both variables are large, that is when slopes are not only steep but long as well.

\section{Hazardous Degrees}

The analysis of the isohytal maps indicates an increase in the rainfall intensities toward the downstream area during winter months (Figure 9). Five flash storms and flood events are recorded (Table 4) and the highest flash flood event occurred in November 1994 (Figures 10, 14, inclusive).

To evaluate the flash floods hazards of El-Atfehy hydrographic basin, Nine morphometric parameters having a direct effect on flooding are analysed. They include the following:

1. Hydrographic basin area (A).

2. Drainage density (Dd).

3. Stream frequency $(\mathrm{F})$.

4. Shape index (Ish).

5. Basin slope $(\mathrm{S})$.

6. Ruggedness number (Rn).

7. Texture ratio (Rt).

8. Weighted mean bifurcation ration (WMRb).

9. Relief ratio $(\mathrm{Rr})$.
They all have direct relationships with the hazards except the weighted mean bifurcation ratio (WMRb), which show an inverse relationship. A hazard scale number from 1 (lowest) to 5 (highest) has been assigned to all parameters. The distribution of the hazard degrees for the studied sub-basins are carried out by,

a. Determination of the minimum and maximum values of each morphometric parameter for the sub-basins.

b. Assessment of the actual hazard degrees for all morphometric parameters, which are located between the minimum and maximum values, which are depending on the empirical relationship between the relative hazard degree of a basin with respect to the flash floods and morphometric parameters.

c. Assuming a straight linear relationship exists between the samples points. The intermediate values can be calculated from the geometric relationship [18].

Hazard degree $=\frac{4(X-X \min )}{(X \max -X \min )}+1$

For the WMRb, which shows an inverse relationship, the hazard degree was calculated using the following equation ${ }^{[18]}$ :

Hazard degree $=\frac{4(X-X \max )}{(X \min -X \max )}+\cdot 1$

Where $\mathrm{X}$ is the value of the morphometric parameters to be assessed for the hazard degree for each sub-basin, $\mathrm{X}_{\min }$ and $\mathrm{X}_{\max }$ are the minimum and maximum values of the morphometric parameters of all sub-basins, respectively. The hazard degree for the study sub-basins of El-Atfehy hydrographic basin is calculated using equations (1) and (2). The total of all hazard degrees for each sub-basin represents the final flood hazard magnitude of El-Atfehy hydrographic basin, (Table 5 \& Fig. 15). The hazard values range from 19.72 in Homary sub-basin, to 30.19 in the Main Channel sub-basin.

\section{Conclusion and recommendations}

The obtained values of the morphometric parameters of the hydrographic drainage network of Wadi El Aftehy hydrographic basin indicate the following results:

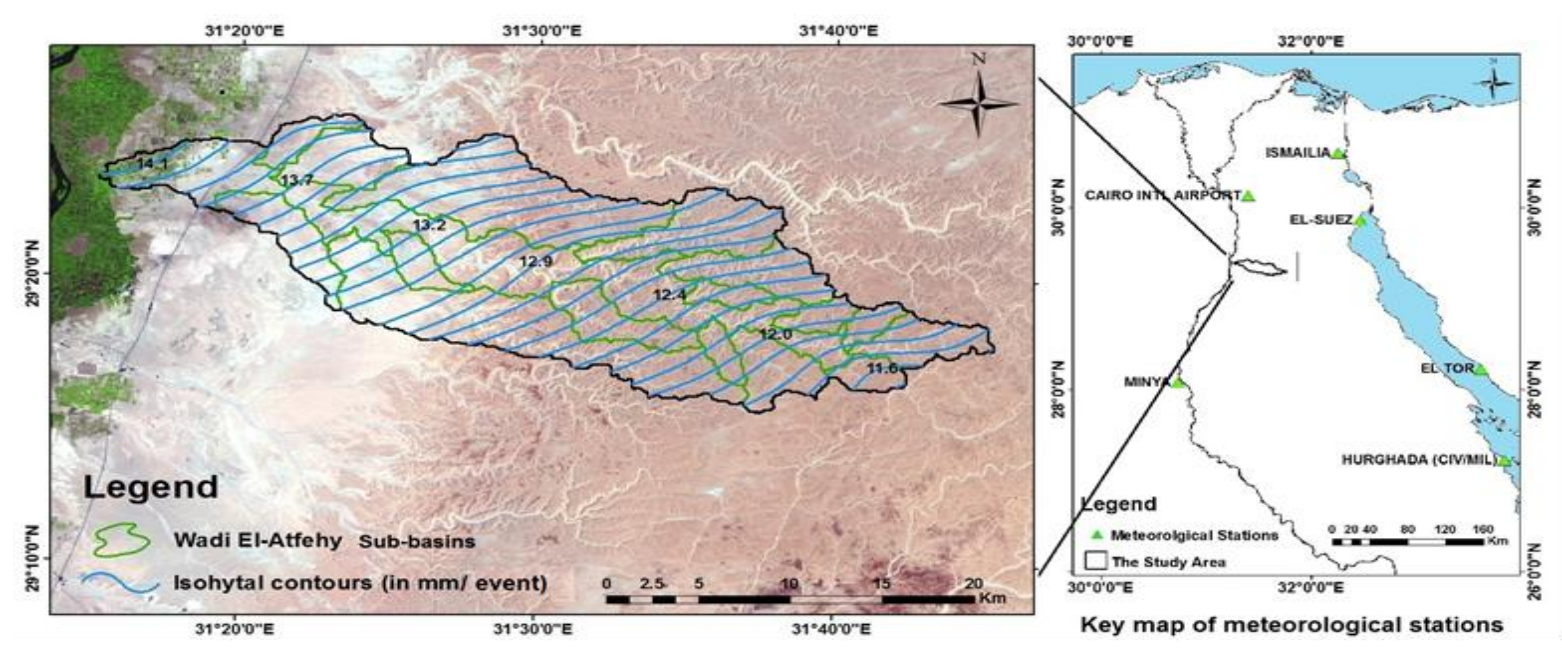

Fig (9): Isohytal map of rainfall intensity of yearly (period: 1984-2002), Wadi El-Atfehy hydrographic basin, Eastern Desert, Egypt. 
1. The linear aspects reveal faster time of arrival of running water from the upstream to the delta as well as high accumulation of surface water into the delta and consequently good chance for groundwater recharge.

2. While areal and relief aspects enhancing high ability for water infiltration and higher potentialities for runoff water harvesting as well as high peaks and flow of shorter duration and faster runoff and therefore flooding is more expected in the basin.

3. Wadi El-Atfehy hydrographic basin has the three classes of the hazard degrees; high, moderate and low. The low hazardous sub-basins include Homary, Subbasin 1, Al- Jibu, Umm Jinays, Abu Mighayir, Umm Ratama, and Umm Shieha, while the moderate hazardous one include Umm Rossa, Sub-basin 2, Sub-basin 3, and Main Channel and the high hazardous include AlHutaliyah, Al- Jarariyah, Abu Mesally, Umm Sayalah and Al-Asliyah sub-basins. The most hazardous part of wadi El-Atfehy hydrographic basin is the middel part including main trunck channel, Al-Jarariyyah, AlHutaliyyah sub-basins.

4. In order to overcome the risks of the flash floods and maximize water harvesting and recharge the groundwater aquifers some retarding rocky dames are recommended for establishing along the main channel especially at the junction sites with the subbasins in the middle stream part and the downstream outlet.

Table 4: Flash flood events over the Eastern Desert of Egypt (After National Water Research Center, Egypt, (NWRC, 2003).

\begin{tabular}{|l|c|c|c|c|c|}
\hline \multicolumn{7}{|c|}{ Flash Flood Events over Eastern Desert (mm) } \\
\hline Ytation & 1987 & 1991 & 1994 & 1996 & 1997 \\
\hline City / Day & $16-$ Oct & 1 -Jan & 2 -Nov & $17-$-Nov & 17 -Oct \\
\hline CAIRO & 0.6 & 4 & 5.2 & 0.1 & 0.2 \\
\hline MINYA & $\ldots$ & $\ldots$ & 0.1 & 4.3 & $\ldots$ \\
\hline EL-TOR & $\ldots$ & $\ldots$ & 6.2 & 15.7 & 0.4 \\
\hline HURGHADA & 13 & 16 & $\ldots$ & $\ldots$ & 0.1 \\
\hline Al-SUEZ & $\ldots$ & $\ldots$ & $\ldots$ & $\ldots .5$ \\
\hline
\end{tabular}

Table 5: Hazard degrees of Wadi El-Atfehy hydrographic sub-basins, Eastern Desert, Egypt.

\begin{tabular}{|l|c|c|c|c|c|c|c|c|c|c|}
\hline sub-basins & $\begin{array}{c}\text { Hazard } \\
\text { degree } \\
\text { Total of } \\
\text { haz. deg. }\end{array}$ & $\begin{array}{c}\text { A } \\
\left(\mathrm{Km}^{2}\right)\end{array}$ & Rt & Ish & F & $\begin{array}{c}\text { Dd } \\
\left(\mathrm{Km}^{-1}\right)\end{array}$ & Rr & Rn & $\begin{array}{c}\text { S } \\
(\mathrm{m} / \mathrm{m})\end{array}$ & WMRb \\
\hline Umm Shieha & 21.4412 & 1 & 2.00 & 2.78 & 5 & 2.25 & 2.77 & 1.07 & 1 & 3.5484 \\
\hline UmmJinays & 20.2741 & 1.00 & 1 & 2.26 & 1 & 1.77 & 5 & 1.79 & 2.1 & 4.2891 \\
\hline Abu Mighayir & 25.3568 & 1.01 & 2.06 & 2.45 & 4.51 & 1.66 & 3.80 & 1.48 & 3.3 & 5 \\
\hline Umm Ratamah & 23.1741 & 1.05 & 1.71 & 2.67 & 2.73 & 2.17 & 3.46 & 1.34 & 3.3 & 4.6394 \\
\hline Sub-basin 1 & 21.6026 & 1.07 & 2.42 & 5 & 3.61 & 2.07 & 3.28 & 1 & 2.1 & 1 \\
\hline Homary & 19.7235 & 1.11 & 1.82 & 1.98 & 3.34 & 4.00 & 2.48 & 1.19 & 1.5 & 2.2331 \\
\hline Abu Mesally & 26.2138 & 1.13 & 2.41 & 2.17 & 4.60 & 5 & 3.99 & 1.35 & 1.6 & 3.8938 \\
\hline Al Asliyyah & 27.2765 & 1.49 & 3.81 & 3.70 & 4.85 & 2.54 & 2.73 & 1.38 & 2.5 & 4.2020 \\
\hline Al Jibu & 24.4964 & 1.52 & 2.80 & 1.57 & 3.21 & 1 & 2.65 & 2.83 & 4.9 & 3.9107 \\
\hline Sub-basin 3 & 23.2724 & 1.57 & 3.44 & 2.05 & 4.81 & 3.65 & 1.14 & 1.22 & 1.4 & 3.9296 \\
\hline Sub-basin 2 & 25.2066 & 1.57 & 4.39 & 2.91 & 4.93 & 3.92 & 1.74 & 1.16 & 1.5 & 3.0117 \\
\hline Umm Roussa & 24.4946 & 1.63 & 2.75 & 2.24 & 2.72 & 1.70 & 2.77 & 2.21 & 4.4 & 3.9900 \\
\hline Umm Sayalah & 27.7164 & 2.03 & 3.04 & 3.14 & 1.96 & 1.54 & 4.61 & 2.99 & 5 & 3.3819 \\
\hline Al Jarariyyah & 29.8056 & 3.60 & 5 & 1.86 & 2.83 & 2.91 & 2.51 & 3.44 & 3.5 & 4.0839 \\
\hline Al Hutilyyah & 28.6590 & 4.00 & 4.55 & 1.53 & 2.92 & 2.56 & 1.74 & 3.64 & 3.3 & 4.3093 \\
\hline Main Channel & 30.1913 & 5 & 2.86 & 1 & 3.54 & 2.46 & 1 & 5 & 4.8 & 4.4263 \\
\hline
\end{tabular}




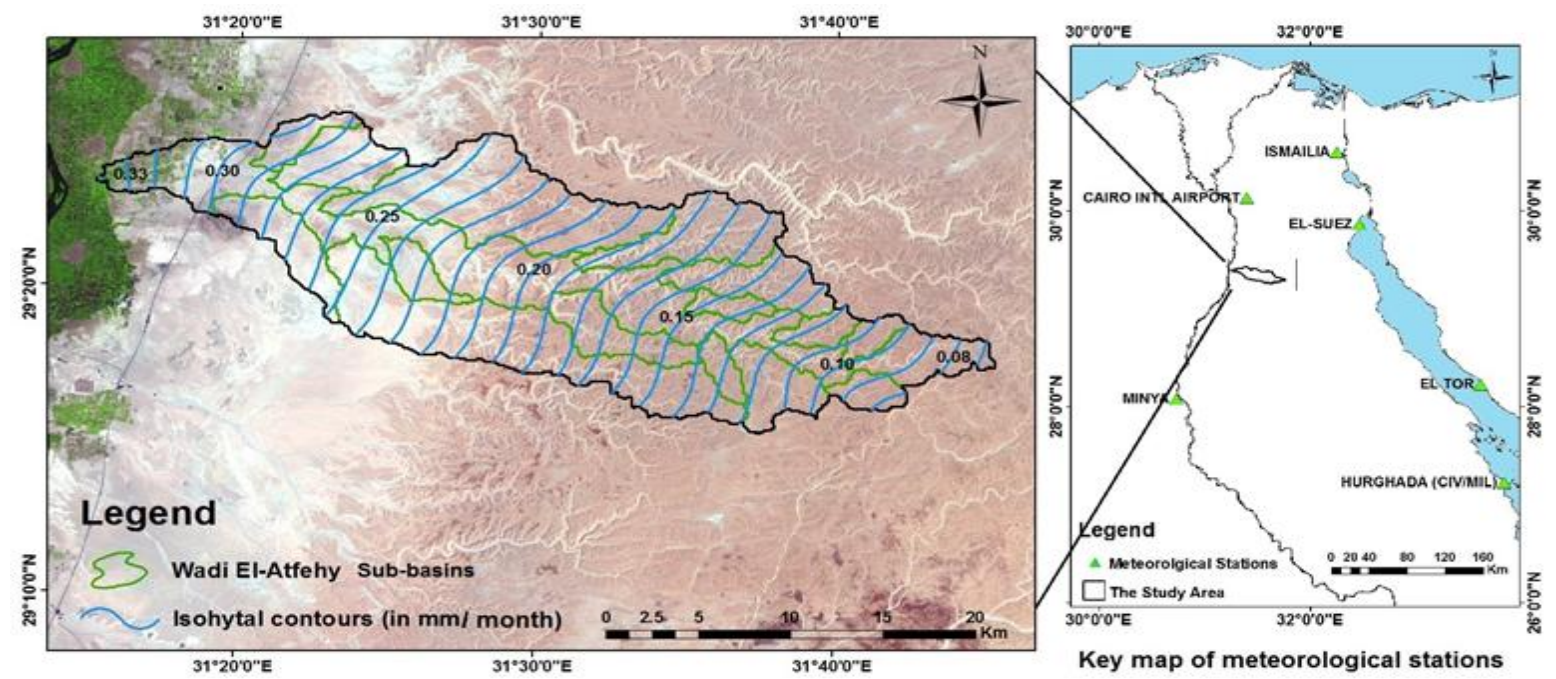

Fig (10): Isohytal map of storm event (17-10-1987), Wadi El-Atfehy hydrographic basin, Eastern Desert, Egypt.
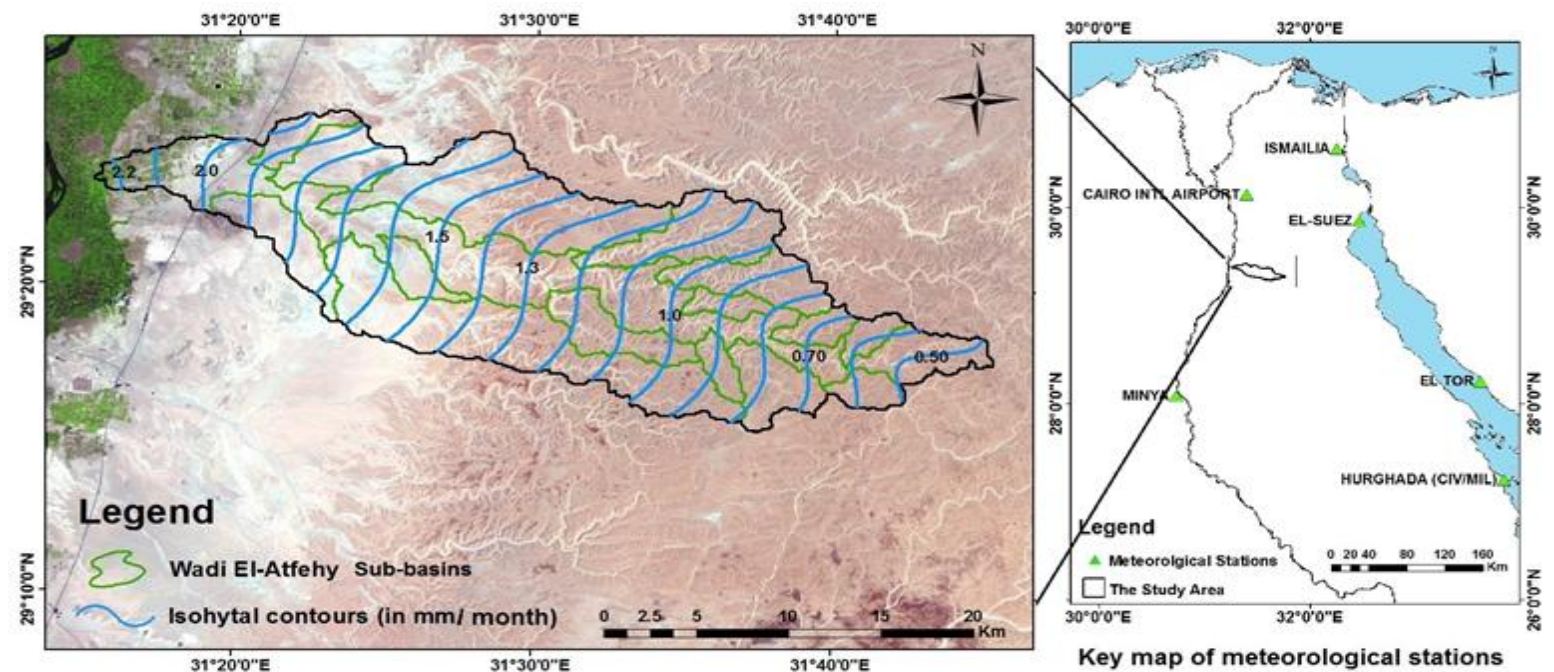

Fig (11): Isohytal map of storm event (1-1-1991), Wadi El-Atfehy hydrographic basin, Eastern Desert, Egypt

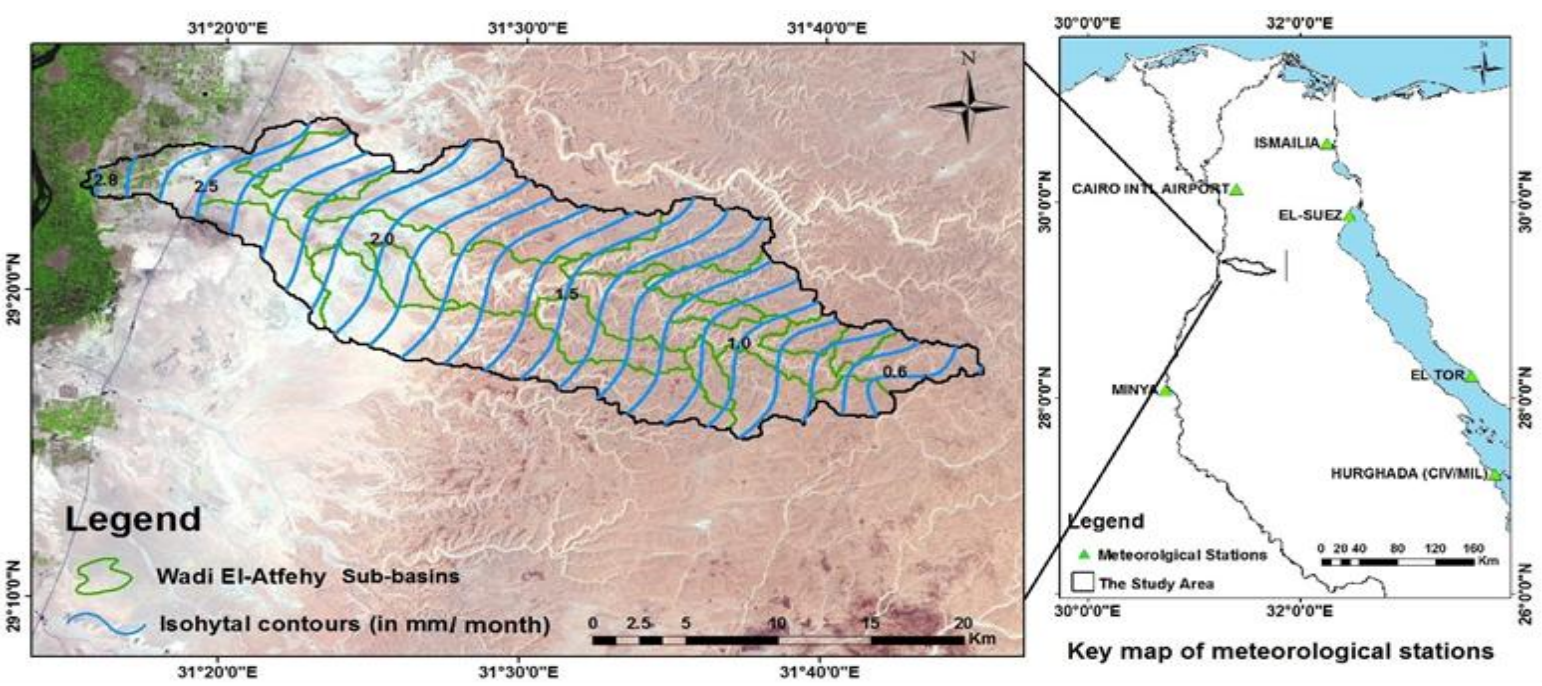

Fig (12): Isohytal map of storm event (2-11-1994), Wadi El-Atfehy hydrographic basin, Eastern Desert, Egypt 

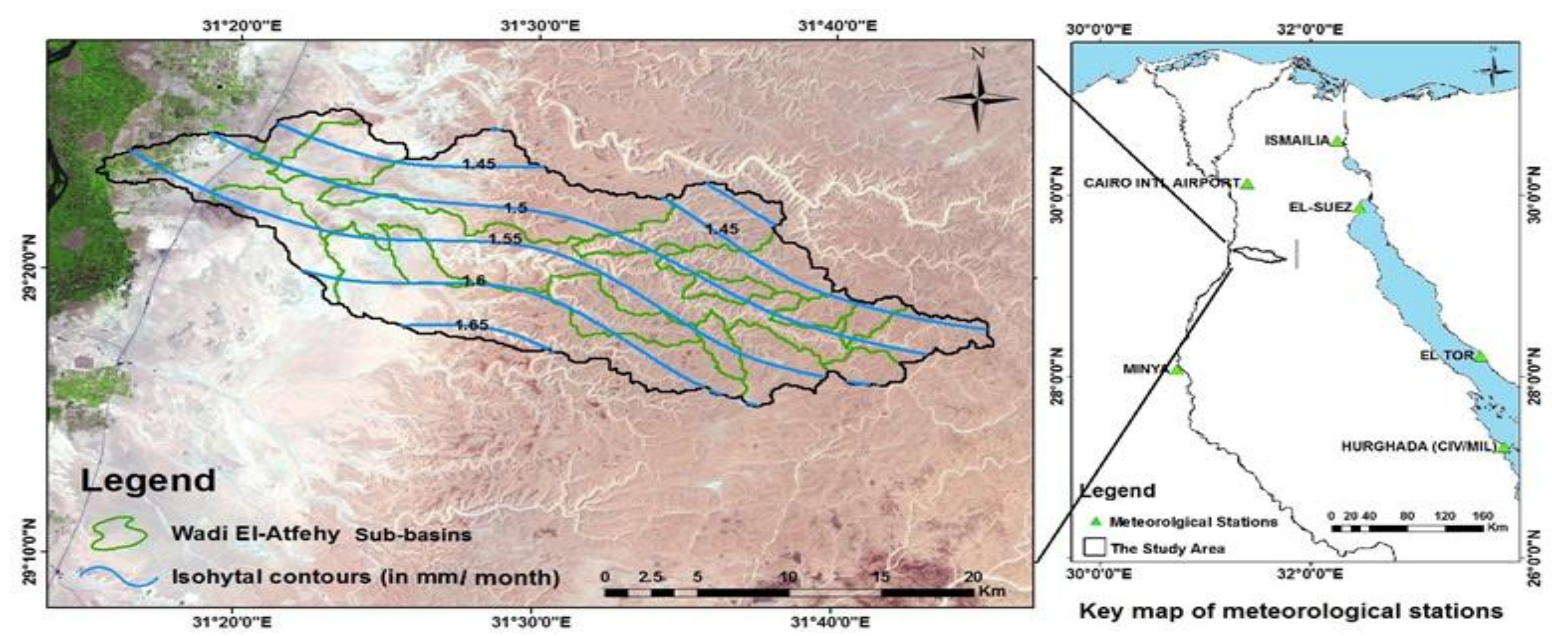

Fig (13): Isohytal map of storm event (17-11-1996), Wadi El-Atfehy hydrographic basin, Eastern Desert, Egypt
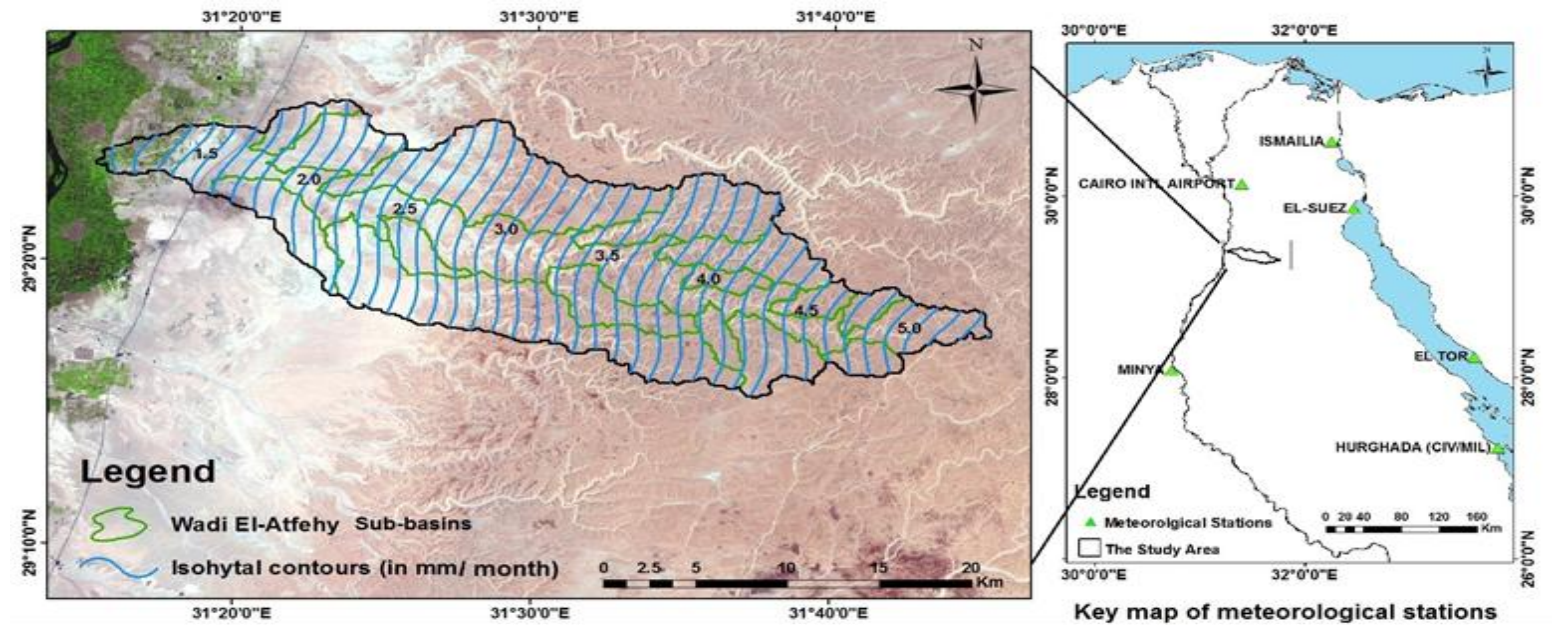

Fig (14): Isohytal map of storm event (17-10-1997), Wadi El-Atfehy hydrographic basin, Eastern Desert, Egypt

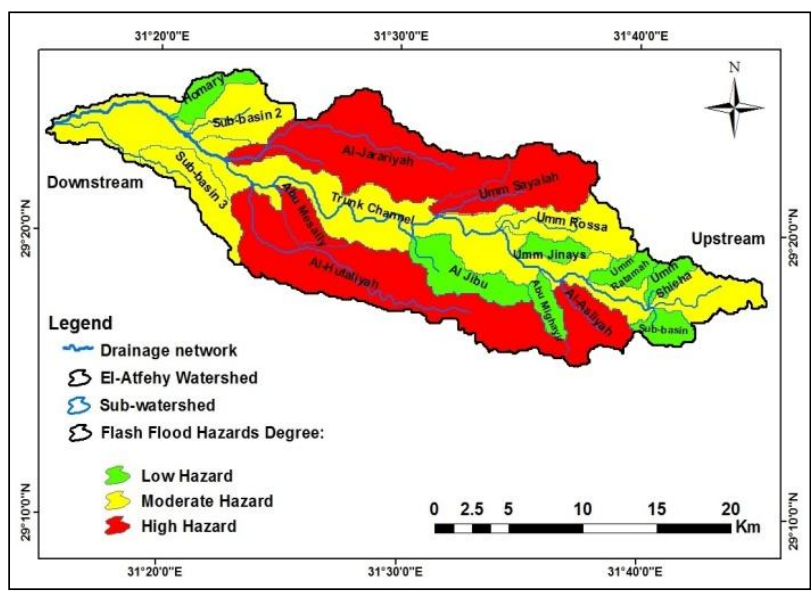

Fig (15): Flash flood hazard degrees of Wadi El-Atfehy hydrographic basin, Eastern Desert, Egypt.

\section{References}

1) Said, R. (1990). The Geology of Egypt, Balkema, Rotterdam, $734 \mathrm{p}$.

2) Korany, E. A. (1995). Hydrology and development of El Atfehy, Eastern Desert, Egypt. $1^{\text {st }}$ Inter. Sci. Conf., Fac. Sci. Azhar Univ., Cairo (Extended Abstract 3p. 15Figs.)
3) Korany, E. A., Soliman, M. M. and Faiad, B. J. (1997). Modeling of the hydrogeologic response of the quaternary aquifer system in the Delta of ElAtfehy, Eastern Desert, Egypt - An Assessment approach for the development of groundwater resources. Ain Shams Sci. Bull., 35: 89-110.

4) Saleh, A. S. (1990). Geomorphological effect of a Torrential flood in El- Atfehy, the Eastern Desert of Egypt. B.S. de Geo. E. Tome LX-III. pp. 99-127.

5) Abdel Moneam, N. A. (2016). Hydrogeological study of water resources and flood control management, Wadi El-Atfehy, Eastern Desert, Egypt. M.Sc. Thesis, Fac. Sci., Ain Shams Univ., 200 p.

6) Suresh, R. (2000). Soil and water conservation engineering, $3^{\text {rd }}$ Ed. 24. Watershed-Concept and Management. pp. 785-813.

7) Horton, R. E. (1945). Erosional development of stream and their drainage basin. Hydrogeological approach to quantitative morphology. Bull. Geol. Soc. Am 56: 275- 370.

8) Gupta, B. L. (1999). Engineering Hydrology, $3^{\text {rd }} \mathrm{Ed}$. Runoff. pp. 46-56.

9) Horton, H. E. (1932). Drainage basin characteristics. Trans. Amer. Geophysics Union 13: 350-361. 
10) Kale, V. and Gupta, A. (2001). Introduction to Geomorphology pp. 84, 85, 86.

11) Schumm, S. A. (1965). Evolution of drainage system and slope in badlands of Perth Amboy, New Jersey. Bull. Geol. Soc. Am. 67: 597-646.

12) Smith, K. G. (1953). Erosional processes and landforms in Badlands National Monument, South Dakota, Nav. Res. Pro. NR 389-042, Tech. Rep. (Columbia Univ. dissertation), $128 \mathrm{p}$.

13) Strahler, A. N. (1957). Quantitative analysis of watershed geomorphology. Trans. Am. Geophysics. Union 38: 913-920.

14) Miller, V. C. (1953). A quantitative geomorphic study of drainage basin characteristic in the clinch, Mountain area, Verdinia and Tennesser, Projet NR 389-042, Tech. Rept.3 Columbia University,
Department of Geology, ONR, Geography Branch, New York.

15) Chopra, R., Dhiman, R. and Sharma, P. K. (2005). Morphometric analysis of sub-basins in Gurdaspur district, Punjab using remote sensing and GIS Technique. Journal of the Indian Society of Remote Sensing, 33(4): 531-539.

16) Faniran (1968). The index of drainage intensity - A prof. new drainage factor. Australian Journal of Science, 31: 328-330.

17) Strahler, A. N. (1964). Quantitative geomorphology of drainage basin and channel network. In: VT Chow (ed.), Handbook of Applied Hydrology McGraw Hill, New York section-4-II.

18) Davis, J. C. (1975). Statics and data analysis in geology. Wiley, New York. 